\title{
Laparoscopic adrenalectomy by transabdominal lateral approach: Should we be afraid of getting started? First 5 years of experience
}

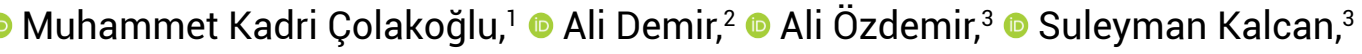 \\ - Gökhan Demiral, ${ }^{3} \odot$ Ahmet Pergel, ${ }^{3} \odot$ Uğur Avcl ${ }^{4}$ \\ 'Department of Gastrointestinal Surgery, Ankara City Hospital, Ankara, Turkey \\ 2Department of General Surgery, Konya Training and Research Hospital, Konya, Turkey \\ ${ }^{3}$ Department of General Surgery, Recep Tayyip Erdoğan University Faculty of Medicine, Rize, Turkey \\ ${ }^{4}$ Department of Internal Medicine, Recep Tayyip Erdoğan University Faculty of Medicine, Rize, Turkey
}

\begin{abstract}
Introduction: A laparoscopic approach has become the standard procedure for removing adrenal masses. However, the need for experience with the technique continues to be emphasized. The aim of this study was to retrospectively review the first 5 years of data of patients who had an adrenal mass and underwent laparoscopic surgery with a transabdominal lateral approach.
\end{abstract}

Materials and Methods: All of the patients at a single institution who were operated on for an adrenal mass using a laparoscopic transabdominal lateral approach between January 2014 and January 2019 were included. The demographic data, any history of other abdominal surgery, American Society of Anesthesiologists score, preoperative diagnosis, hormonal characteristics of the tumor, intraoperative parameters, intraand postoperative complications, and the histological diagnosis were analyzed.

Results: A total of 42 laparoscopic adrenalectomy procedures were performed. The mean age of the patients was $50.64 \pm 13.22$ years. The lesion was located on the right side in $52.3 \%$ of the patients. Nine patients $(21.4 \%)$ had previously undergone abdominal surgery for various reasons. The majority of the adrenal masses were a secretory adenoma or hyperplasia of the gland. Four patients were operated on for metastasis of other malignancies and others were non-secreting tumors. The mean size of the adrenal lesions was $29.4 \pm 12.7 \mathrm{~mm}$. The mean operative time was 140.45 minutes and the mean blood loss was $62.9 \mathrm{~mL}$. Intraoperative complications occurred in 3 cases (7.1\%). Complications were observed postoperatively in 9 patients $(21.4 \%)$. The mean length of hospitalization was $3.8 \pm 2.1$ days.

Conclusion: A laparoscopic adrenalectomy proved to be a safe and feasible method, even in cases in which it was the operator's first experience with the procedure. The results of a first application were similar to those reported in the literature. However, we strongly believe that other prior laparoscopic experience is required to achieve these results.

Keywords: Adrenalectomy; laparoscopy; transabdominal. 


\section{Introduction}

Adrenal glands are endocrine glands located on the superior-medial side of the kidneys in the retroperitoneal area on both sides. Surgical removal of this gland or both glands is needed due to various endocrine diseases, masses, metastasis of different organs or malignancies. The surgical treatment of the adrenal gland can be challenging that it is located in the retroperitoneal area, dissection of the adipose tissue could be difficult especially in obese patients, it has close relationship with the main vascular structures (aorta, vena cava) and high risk of bleeding because of the fragile, and highly vascularized nature of the organ.

There are different ways to reach the adrenal gland surgically (open, laparoscopic, robotic). Each method has defined advantages and disadvantages. ${ }^{[1]}$ The most commonly used method is laparoscopy and can be applied from the transabdominal or retroperitoneal area by anterior, lateral or posterior approach. ${ }^{[2]}$ Although many studies comparing transabdominal and retroperitoneal approaches have shown different advantages, transabdominal approach is mostly preferred because it provides a large area in the abdomen, allows excision of medium size $(<6 \mathrm{~cm})$ masses and is easy to teach ${ }^{[1,3]}$ but there is still a debate about laparoscopic approach for large $(>6 \mathrm{~cm})$ or malignant masses. ${ }^{[4-6]}$

The laparoscopic approach to adrenal masses has now become the standard procedure, and with the widespread use of laparoscopy, its use has increased rapidly. However, it is emphasized that it should still be performed in experienced centers because of its surgical risks. But to become an experienced center, first, you need to start. The aim of this study is to retrospectively review the first 5 years of data of patients with adrenal masses, undergone laparoscopic transabdominal lateral approach in an academic referral center and evaluate safety and outcomes of the procedure. The collected data and the results was compared with the literature.

\section{Materials and Methods}

Between January 2014 and January 2019, all patients who were operated by laparoscopic transabdominal lateral approach in a university hospital were included in the study. Ethics committee approval was not obtained due to the retrospective nature of the study. Patient files, surgical records, pathology results and digital datas were analyzed retrospectively. Demographic datas, history of preoperative abdominal surgery, American Society of Anesthesiologists (ASA) scores, preoperative diagnosis, hormonal characteristics of adrenal tumor, intraoperative parameters, intra- and postoperative complications, outcomes and histological diagnosis were analyzed. Only, patients suspected to have primary adrenal malignancy were excluded.

\section{Preoperative Evaluation}

All of the patients were evaluated preoperatively by an endocrinologist and the surgical decision was made after consultation in the endocrine council of the hospital which is consisted of endocrinologists, radiologists and surgeons. In addition, medical oncologist's opinion was obtained for patients with a different organ malignancy and adrenal metastasis. All of the hormonal tests of the patients (female or male sex hormones, thyroid function tests, plasma adrenocorticotrophic hormone [ACTH], cortisol, androstenedione, dihydroepiandrosterone sulfate (DHEA-S), 17- $\alpha$-hydroxy progesterone, aldosterone, plasma renin activity, metanephrine, normetanephrine, urine catecholamines [adrenaline, noradrenaline, vanilmandelic acid]) were evaluated by the endocrinologist and dexamethasone suppression test was applied when necessary.

In patients with functional adrenal masses, the blood pressure of the patients was controlled by medications (alpha-1 blockers), which were started 2 weeks before surgery and they were hydrated by intravenous way preoperatively. Also preoperative preparation was done by spironolactone and potassium for Conn's syndrome and anticortisolic agents for Cushing's syndrome if necessary. Abdominal computed tomography (CT) was performed in all patients and magnetic resonans imaging (MRI) and [18F] fluorodeoxyglucose positron emission tomography (FDG-PET/CT) examinations were added if necessary.

\section{Surgical Procedure}

All surgical procedures were performed by the same surgical team specialized in minimal invasive surgery. All patients received general anesthesia with orotracheal intubation and antibiotic prophylaxis (cefazoline $2 \mathrm{~g}$ i.v.) was performed. No nasogastric or urinary catheters placed before surgery. All patients positioned in the lateral decubitus position with an inclination of $50-60^{\circ}$ relative to the operating table according to the side of the lesion. The brachial plexus was protected with an axillary pad 
and the arms were fixed. Veress needle was used to create pneumoperitoneum even for the patients with history of abdominal surgery. Pneumoperitoneum was maintained at $14 \mathrm{mmHg}$ by insufflation of carbon dioxide $\left(\mathrm{CO}_{2}\right)$. After diagnostic laparoscopy performed, if the stomach was dilated, it was aspirated with an orogastric catheter and then the catheter was removed. The classic form of laparoscopic adrenal surgery is described by Gagner et al. ${ }^{[7]}$ (1992) and current surgical technique was described by Hamilton et al. ${ }^{[8]}$ (2001) and all operations were performed in this manner. Dissections were always done with hook and electrothermal bipolar vessel sealing device (LigaSureTM vessel-sealing system, Medtronics, Minneapolis, USA). Major vascular structures were obliterated with metallic clips. Direct contact with the adrenal gland was avoided during the operation. Manipulation was performed only with laparoscopic peanut dissector. Surgical specimens were positioned in endo-bag and removed through the extended $10 \mathrm{~mm}$ trocar place. One closed suction drain was placed in all patients.

\section{Left Adrenalectomy}

Three trocars (two $10 \mathrm{~mm}$ and one $5 \mathrm{~mm}$ ) were used for the left adrenalectomy operation. Extra trocar was not avoided if needed. The first step is mobilization of the splenic flexure of the colon and exposing Gerota's fascia. With the effect of the patient's position and gravity, the colon moves away from the area and after that the connections at the inferior-posterior of the spleen are mobilized. The spleen does not need to be completely released. The peritoneum between the colon and spleen is dissected and the tail portion of the pancreas is displayed and the avascular areolar tissue between the pancreas and Gerota's fascia is divided. At this point, especially in obese patients, damage to pancreatic tissue should be avoided. Adrenal gland should be approached medially. The first lateral dissection causes the gland to overturn medially by gravity and makes it difficult to dissect the vascular structures. Dissection started at the superomedial border of the adrenal gland by the diaphragm and continued toward the renal hilum. Here, the dissection plan can sometimes be difficult and the fourth trocar may be needed for adequate retraction. Adrenal vein is identified and skeletonized at its confluence with the inferior phrenic vein. In some extreme cases, it may also be necessary to visualize the renal vein to be sure. The adrenal vein is dissected after double clipping and the adrenal gland is separated and removed via the left kidney and lateral attechments with the aid of an energy device.

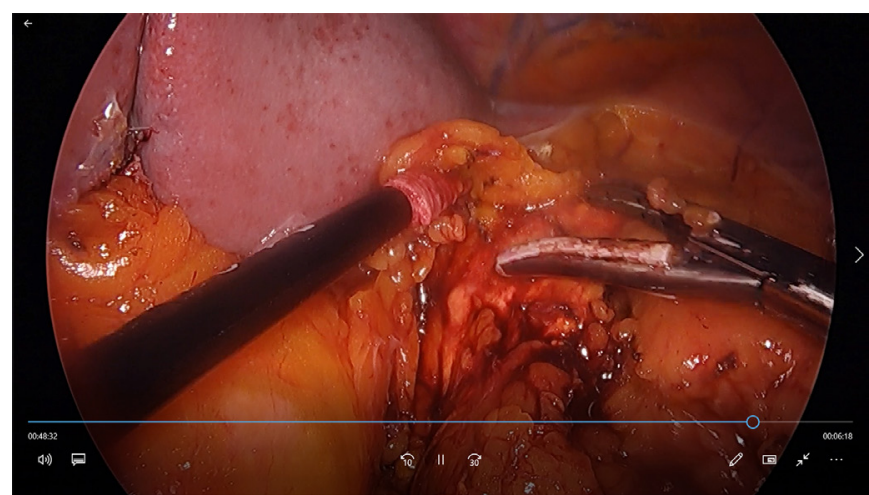

Figure 1. Left adrenalectomy.

\section{Right Adrenalectomy}

Four trocars (two $10 \mathrm{~mm}$ and two $5 \mathrm{~mm}$ ) were used for the right adrenalectomy operation. The fourth trocar is often required for retraction of the liver, unlike left adrenalectomy. Extra trocar was not avoided if needed. After the liver retractor has been placed the first step is dividing the right triangular ligament of the liver, and continuing medially to the inferior vena cava (IVC) to expose and divide Gerota's fascia. During dissection, the retraction of the adrenal gland following retraction of the liver provides relief. Dissection is carried along the superomedial edge of the adrenal gland toward the lateral edge of the IVC to mobilize the medial aspect of the adrenal gland. At this point, care should be taken not to bleed or to lose the plan, especially in bulky tumors. The right adrenal vein is very short and encountered just inferior to where the IVC enters the liver. The adrenal vein should be carefully skeletonized and cut after double clipping. The adrenal gland is separated and removed via the right kidney and lateral attechments with the aid of an energy device.

\section{Postoperative Management}

In the postoperative period, only patients who had to be followed up due to their comorbidities were taken to the intensive care unit. Most of the patients were followed up in the clinic. Oral feeding and mobilization initiated on the first postoperative day. Abdominal drains were removed on the first or second operation day if there is no sign of bleeding. Postoperative medication in hormonal active patients was arranged according to the recommendations of the endocrinologist. Follow-up after discharge was performed by both surgeon and endocrinologist.

An intraoperative complication was defined as any undesirable event describe in the operative report and a postoperative complication was defined as an undesirable 
event happened in the postoperative course. All of the complications were recorded and graded according to the Dindo-Clavien-grade scale. ${ }^{[9]}$

\section{Statistical Analysis}

Statistical analysis were performed with SPSS 20.0 (SPSS Inc., Arlington, Virginia). Continuous variable was expressed as the mean \pm SD (min-max). Categorical Data were compared with Chi-square analysis and Pearson correlation analysis and Student t-test were used to evaluate the correlation between variables. A p value $<0.05$ was considered statistically significant.

\section{Results}

During the study period, a total of 42 laparoscopic adrenalectomy procedures by transabdominal lateral approach were performed and the vast majority of cases were women $(\mathrm{n}=33,78.6 \%)$. The mean age of all patients included to the study was $50.64 \pm 13.22$ (range 14-76) years old. The lesion was located on the right side in $52.3 \%$ of the patients. Nine patients (21.4\%) had previously undergone abdominal surgery for various reasons (One right hemicolectomy, one subtotal gastrectomy, one cholecystectomy, four cesarean sections, two appendectomies). The majority of adrenal masses were secretory adenoma or hyperplasia of the gland (Pheochromocytoma/ Cush-
ing/Hyperaldosteronism, $\mathrm{n}=32,76.1 \%)$. Four patients were operated for metastasis of other malignancies (one endometrial cancer, one lymphoma and two neuroendocrine tumors) and others were non-secreting tumors. Mean size of adrenal lesions was $29.4 \pm 12.7 \mathrm{~mm}$ (range 6-80 $\mathrm{mm})$. Demographic characteristics and ASA scores of patients, side of the lesions and indications for operations are presented in Table 1 .

Mean operative time was 140.45 min (range 69-340). Pearson correlation analysis was used to determine whether there was a correlation between operation time and tumor size but no correlation was found $(\mathrm{p}=0.732)$. Also no correlation was found between operation time and gender ( $\mathrm{p}=0.778$ ), tumor location (left/rigt, $\mathrm{p}=0.984$ ) and preoperative diagnosis $(\mathrm{p}=0.166)$. There was a borderline relationship between operation time and age $(\mathrm{p}=0.046)$. Operation time was prolonged as the patient's age increased. Mean blood loss was $62.9 \mathrm{ml}$ (range 10-180). No patient required blood transfusion intraoperatively.

Intraoperative complications occured in three cases (7.1\%) and both complications belong to the beginning part of the operation. In one patient, minor bleeding occured due to stinging of the veress needle into the right lobe of the liver and this bleeding was controlled by simple cauterization. In one patient who had left adrenalectomy, the first trocar was penetrated into the descending colon due

Table 1. Demographic characteristics of patients and indications for operation

\begin{tabular}{lccc} 
& Number $(\mathbf{n})$ & Percent $(\%)$ & Mean age (years) \\
\hline Gender & 33 & & $49.6(14-76)$ \\
$\quad$ Female & 9 & 78.6 & $54.3(43-70)$ \\
$\quad$ Male & 1 & 21.4 & \\
ASA score & 14 & 3.4 & \\
1 & 21 & 50 & \\
2 & 6 & 14.3 & \\
3 & & 7.1 & \\
4 & 3 & 14.3 & \\
Previous abdominal surgery & 6 & 23.8 \\
$\quad$ Upper abdomen & & 38 \\
Lower abdomen & 10 & 14.3 \\
Indications for operation & 16 & 14.3 \\
Pheochromocytoma & 6 & 9.5
\end{tabular}


to intraabdominal adhesions. This perforation area was sutured laparoscopically and operation was continued. No major vascular injury was seen in any of the patients. Only one patient had intraoperative hypertensive crisis (youngest patient, 14 years old) and was treated successfully without any postoperative injuries. There was no conversion to open surgery.

There were no major complications while minor complications were observed in nine patients $(21.4 \%)$ postoperatively. Five patients had postoperative ultrasonography for pain, four had hematoma and one had fluid collection. Only one of these patients required percutaneous drainage. Other patients were treated conservatively. One patient developed abdominal wall hematoma due to bleeding from the trocar site and was followed conservatively. No postoperative blood transfusion was required. Two patients developed infecton in the enlarged trocar field and one patient developed fever secondary to atelectasis. These three patients were treated medically. According to Clavien Dindo Classification number of patients were Grade I: 5, Grade II: 3 and Grade 3: 1 . No mortality was observed.

The mean hospitalization day was $3.8 \pm 2.1$ (range 1-10). The controls of the patients after discharge were provided by endocrinologist and the surgeon in outpatient clinic. Surgery determined an hormonal serum levels normalization in all patients with functional adenoma in near-term follow-up. Operative and postoperative datas are presented in Table 2.

\section{Table 2. Operative and postoperative datas}

\begin{tabular}{lc} 
& $\begin{array}{c}\text { Laparoscopic } \\
\text { transperitoneal } \\
\text { adrenalectomy } \\
\text { (n=42) }\end{array}$ \\
\hline Mean size of tumor (mm) & $29.4 \pm 12.7 \mathrm{~mm}$ \\
Mean operative time (minutes) & $140.45 \mathrm{~min}$ \\
Mean blood loss (ml) & $62.9 \mathrm{ml}$ \\
Complications (\%) & \\
Intraoperative & $7.1 \%$ \\
Postoperative & $21.4 \%$ \\
Clavien-Dindo Grade I & $11.9 \%$ \\
Clavien-Dindo Grade II & $7.1 \%$ \\
Clavien-Dindo Grade III & $2.3 \%$ \\
Mean hospital stay (day) & $3.8 \pm 2.1 \mathrm{days}$
\end{tabular}

\section{Discussion}

Laparoscopic adrenalectomy is still considered as a safe but difficult surgical procedure and emphasized that it should be performed in experienced centers. This makes surgeons cautious about the procedure. Traditionally, rigth-sided, large-sized or hormonal active tumors are believed to be more challenging but some recent publications claim otherwise. ${ }^{[6,10,11]}$ More interestingly, Limberg et al. ${ }^{[12]}$ reported that laparoscopic adrenalectomy is as safe and feasible as laparoscopic cholecystectomy procedure and emphasized that physicians should not hesitate to refer their patients to surgery. Herewith, on the basis of our experience in minimally invasive surgery, we aimed to demonstrate the applicability of the procedure by representing our 5-year results.

From the first description of Gagner et al., laparoscopic adrenalectomy is now the standard procedure for adrenal lesions. Although no comparison was made with open technique in this study, its superiority over open technique has been shown in many studies in the literature. ${ }^{[13-15]}$ In particular, magnification provided by laparoscopy allows surgeons to perform anatomical dissection more safely and minimizes complications. In addition, the traditional advantages of laparoscopy creates superiority to open surgery with less pain, early mobilization and less scarring. However, the close relationship between adrenal glands with large venous structures, being a fragile organ that is prone to bleeding, as well as working in a narrow retroperitoneal space complicates the procedure and intimidate surgeons. Although we think that this kind of hesitations may be in any kind of laparoscopic procedure, we strongly argue that it is necessary to know the anatomy of the region and to have laparoscopic experience before starting minimally invasive adrenalectomy.

The study included all consecutive surgical procedures and it can be seen from the demographic characteristics of the patients that all patient groups (age, diagnosis, location, tumor size) were taken into consideration. Only the female population dominates the balance. The intraoperative and postoperative results of our study are consistent with many studies showing the applicability of transabdominal lateral adrenalectomy in the literature. ${ }^{[16-19]}$

Although there are publications advocating otherwise, it is emphasized that obesity, abdominal surgery history, tumor localization (right-left), patient comorbidities and pheochromocytoma diagnosis may change the opera- 
tion results especially the operation time. ${ }^{[20]}$ The mean operation time was 140.45 minutes in our study and was consistent with the literature. No relationship was found between these factors and operation time, however, body mass index could not be included due to lack of data. Mean blood loss is also consistent with the literature. ${ }^{[19]}$

Park et al. ${ }^{[17]}$ reported that the complication rates $(18.3 \%$ vs $11.3 \%$ ) and hospital stay (5.5 vs 3.9 days) of low-volume surgeons were higher. This study is noteworthy, but it is difficult to evaluate complication rates as there is no standardized definition of complications in general. In our study, postoperative complication rates were found to be $21.4 \%$, but half of them were Clavien-Dindo Grade I which were conservatively approached. The total of Grade II and III complications were $9.4 \%$, which was consistent with other studies. The average hospital stay of 3.8 days is a confirmation of this situations, which is consistent with other studies. The study of Gallagher et al. ${ }^{[18]}$ confirms this in their study reflecting no correlation between low-volume surgeons and complication rates or length of hospital stay.

Conversion to open surgery and mortality were not observed in our study. However, we believe that this is not suitable for evaluation because of the low number of cases. Retrospective design of the study, female gender predominance and lack of body mass index data are the limitations of the study. In addition, although early results show that surgery is successful, lack of late results is one of the limitations of the study. In addition noone should ignore that the frequency of the factors that make laparoscopic adrenalectomy difficult to be found less in these 42 cases.

\section{Conclusion}

Laparoscopic adrenalectomy is a safe and feasible method even in the first experience. The results of the first application are similar to the literature. However, we strongly believe that laparoscopic experience is required to achieve this results.

\section{Disclosures}

Financial support: The authors declared that this study has received no financial support.

Peer-review: Externally peer-reviewed.

Conflict of Interest: None declared.

\section{References}

1. Madani A, Lee JA. Surgical Approaches to the Adrenal Gland. Surg Clin North Am 2019;99:773-91. [CrossRef]

2. Conzo G, Tartaglia E, Gambardella C, Esposito D, Sciascia V, Mauriello $C$, et al. Minimally invasive approach for adrenal lesions: systematic review of laparoscopic versus retroperitoneoscopic adrenalectomy and assessment of risk factors for complications. Int J Surg 2016;28:S118-23. [CrossRef]

3. Conzo G, Gambardella C, Candela G, Sanguinetti A, Polistena A, Clarizia G, et al. Single center experience with laparoscopic adrenalectomy on a large clinical series. BMC Surg 2018;18:2. [CrossRef]

4. Pisano G, Calò PG, Erdas E, Pigliaru F, Piras S, Sanna S, et al. Adrenal incidentalomas and subclinical Cushing syndrome: indications to surgery and results in a series of 26 laparoscopic adrenalectomies. Ann Ital Chir 2015;86:406-12.

5. Serji B, Souadka A, Benkabbou A, Hachim H, Jaiteh L, Mohsine $\mathrm{R}$, et al. Feasibility and safety of laparoscopic adrenalectomy for large tumours. Arab J Urol 2016;14:143-6. [CrossRef]

6. Mohammed A, Amine H, Atiq SE, Mohammed B, Ouadii $M$, Khalid $M$, et al. Applicability and outcome of laparoscopic adrenalectomy for large tumours. Pan Afr Med J 2018;31:23. [CrossRef]

7. Gagner M, Lacroix A, Bolté E. Laparoscopic adrenalectomy in Cushing's syndrome and pheochromocytoma. N Engl J Med 1992;327:1033. [CrossRef]

8. Hamilton BD. Transperitoneal laparoscopic adrenalectomy. Urol Clin North Am 2001;28:61-70. [CrossRef]

9. Dindo D, Demartines N, Clavien PA. Classification of surgical complications: a new proposal with evaluation in a cohort of 6336 patients and results of a survey. Ann Surg 2004;240:205-13. [CrossRef]

10. Kokorak L, Soltes M, Vladovic P1, Marko L. Laparoscopic left and right adrenalectomy from an anterior approach - is there any difference? Outcomes in 176 consecutive patients. Wideochir Inne Tech Maloinwazyjne 2016;11:268-73. [CrossRef]

11. Öz B, Akcan A, Emek E, Akyüz $M$, Sözüer $E$, Akyıldız $H$, et al. Laparoscopic surgery in functional and nonfunctional adrenal tumors: a single-center experience. Asian J Surg 2016;39:137-43. [CrossRef]

12. Limberg J, Ullmann TM, Gray KD, Stefanova D, Zarnegar R, Li $J$, et al. Laparoscopic Adrenalectomy has the Same Operative Risk as Routine Laparoscopic Cholecystectomy. J Surg Res 2019;241:228-34.

13. Prinz RA. A comparison of laparoscopic and open adrenalectomies. Arch Surg 1995;130:489-92. [CrossRef]

14. Hallfeldt KK, Mussack T, Trupka A, Hohenbleicher F, Schmidbauer S. Laparoscopic lateral adrenalectomy versus open posterior adrenalectomy for the treatment of benign adrenal tumors. Surg Endosc 2003;17:264-7. [CrossRef]

15. Lee J, El-Tamer M, Schifftner T, Turrentine FE, Henderson WG Khuri S, et al. Open and laparoscopic adrenalectomy: analysis of the National Surgical Quality Improvement Program. J Am Coll Surg 2008;206:953-9. [CrossRef] 
16. Economopoulos KP, Phitayakorn R, Lubitz CC, Sadow PM, Parangi S, Stephen $A E$, et al. Should specific patient clinical characteristics discourage adrenal surgeons from performing laparoscopic transperitoneal adrenalectomy? Surgery 2016;159:240-8. [CrossRef]

17. Park HS, Roman SA, Sosa JA. Outcomes from 3144 adrenalectomies in the United States: which matters more, surgeon volume or specialty? Arch Surg 2009;144:1060-7.

18. Gallagher SF, Wahi M, Haines KL, Baksh K, Enriquez J, Lee
$\mathrm{TM}$, et al. Trends in adrenalectomy rates, indications, and physician volume: a statewide analysis of 1816 adrenalectomies. Surgery 2007;142:1011-21. [CrossRef]

19. Coste T, Caiazzo R1, Torres F1, Vantyghem MC2, Carnaille B1, Do Cao C, et al. Laparoscopic adrenalectomy by transabdominal lateral approach: 20 years of experience. Surg Endosc 2017;31:2743-51. [CrossRef]

20. Raffaelli M, De Crea C, Bellantone R. Laparoscopic adrenalectomy. Gland Surg 2019;8:S41-S52. [CrossRef] 